\title{
STUDYING LUMP SOLUTIONS, ROGUE WAVE SOLUTIONS AND DYNAMICAL INTERACTION FOR NEW MODEL GENERATING FROM LAX PAIR
}

\author{
M. K. ElboreE*
}

\begin{abstract}
In this paper, we consider the $(3+1)$-dimensional Burgers-like equation which arises in fluid mechanics, which constructed from Lax pair generating technique. The bilinear form for this model is obtained to construct the multiple-kink solutions. Lump solution, rogue wave solutions are constructed via the obtained bilinear form for this model. The physical phenomena for these solution are analyzed by studying the influence of the parameters for these solutions. The phase shifts, propagation directions and amplitudes for these solutions are controlled via these parameters. The collisions between the lump wave and the stripe soliton, which is called lumpoff solution are completely non-elastic interaction. Finally, the figures of the solutions are shown to study the dynamical behavior for the lump, rogue wave and the properties of the interaction phenomena under various parameters for $(3+$ 1)-dimensional Burgers-like equation. These results can't be found in the previous scientific papers.
\end{abstract}

Mathematics Subject Classification. 35Q51, 35Q53, 35C99, 37K40, 33F10.

Received April 18, 2020. Accepted July 5, 2020.

\section{INTRODUCTION}

Lax pair generating technique is a vigorous tool to construct integrable equations in $(2+1)$ dimensions [3].

Soliton solutions for nonlinear partial differential equations (NLPDEs) are an important great significance in nonlinear science such as rational solutions $[6,38]$. Also solitons can be generated via Lie group analysis [7]. Symbolic computation on an observationally/experimentally-supported (2+1)-dimensional generalized variablecoefficient Kadomtsev-Petviashvili-Burgers-type equation is done in [9].

Lump solutions are localized and decay algebraically $[8,14,26]$ and a special kind of rational solutions. Hirota bilinear method [20] is one of the approaches to study the lump solutions. Lump solutions are completely elastic for some integrable NLPDEs [21, 28, 34] and others are completely inelastic [33, 36, 39]. Some examples of lump solutions for some integrable equations are found such as Ishimori I equation [16] and $(2+1)$-dimensional extended KP equation [27].

Rogue wave solution was used to describe the momentous disastrous ocean waves [10] and can lead to devastate impact to the navigation. Darboux transformation [5, 32], auto- and non-auto-Bácklund transformations [11], bilinear method [4] are ued to obtain the rogue wave solution.

Keywords and phrases: Bilinear formalism, lump solitons, rogue wave solutions, $(3+1)$-dimensional Burgers-like equation, lump-stripe soliton interaction.

Mathematics Department, Faculty of Science, South Valley University, Qena, Egypt.

* Corresponding author: mkelboree@gmail.com 
Interaction solutions occur between lump solutions and one stripe [17, 22-25, 29] or line soliton based on Hirota bilinear form and combining the rational quadratic functions (exponential functions) were analyzed in $[15,31]$, such as $(4+1)$-dimensional Fokas and $(3+1)$-dimensional potential Yu-Toda-Sasa-Fukuyama equation [30].

The dates of Burgers' equation back to 1915 when Bateman derived it in a physical context [1] and concluded its form as a model in the theory of turbulence [2]. This model appears as a simplification of more complex model and used to understand some the behavior of the general problem such as Navier Stokes equations simplification and Traffic Flow. This model also is the lowest order approximation for one-dimensional propagation for weak shock waves in a fluid and one of the fundamental model equations in fluid mechanics [35].

In this work, we derive the bilinear form for $(3+1)$-dimensional Burgers-like equation in Section 2, based on the bilinear formalism, lump solitons were obtained in Section 3 and rogue wave solutions are derived for $(3+$ 1)-dimensional Burgers-like equation in Section 4. Also, the interaction between a lump soliton and one stripe for the above model was constructed in Section 5. Finally, discussions and conclusions were given in Section 6 . Remark: The results obtained in this paper for $(3+1)$-dimensional Burgers-like equation are new and can't be found in any previous papers.

Via the idea presented in [3] for the Lax pair generating function presented to develop $(3+1)$-dimensional $\mathrm{KdV}$ like equation, in a like manner the $(3+1)$-dimensional Burgers-like equation are constructed in [36]

$$
\begin{aligned}
& u_{t}+\alpha\left(2 u u_{x}+u_{x x}\right)+\beta\left(u u_{y}+u_{x} v+u_{x y}\right)+\gamma\left(u u_{z}+u_{x} w+u_{x z}\right)=0, \\
& v_{x}=u_{y}, \\
& w_{x}=u_{z},
\end{aligned}
$$

where $\alpha, \beta$ and $\gamma$ are real parameters. if $\gamma=0$, it can be noted as equation (1.1) converts to a $(2+1)$ - dimensional Burgers-like equation and if $\beta=\gamma=0$, the equation (1.1) can be converted to the classical Burgers equation.

\section{Bilinear FORM AND SOlitons SOlutions FOR $(3+1)$-Dimensional BURGERS-LIKE EQUATION}

Under the dependent variable transformation

$$
\begin{aligned}
& u(x, y, z, t)=(\ln f(x, y, z, t))_{x}+u_{1} \\
& v(x, y, z, t)=(\ln f(x, y, z, t))_{y}+v_{1} \\
& w(x, y, z, t)=(\ln f(x, y, z, t))_{z}+w_{1}
\end{aligned}
$$

where $u_{1}, v_{1}$ and $w_{1}$ are constants will be determined later.

The Hirota bilinear form $[12,18,19]$ for equation (1.1) can be deduced as follows

$$
\begin{aligned}
& \left(2 D_{x} D_{t}+2 \alpha\left(D_{x}^{3}+2 u_{1} D_{x}^{2}\right)\right. \\
& \left.\quad+\beta\left(2 D_{x}^{2} D_{y}+2 v_{1} D_{x}^{2}+2 u_{1} D_{x} D_{y}\right)+\gamma\left(2 D_{x}^{2} D_{z}+2 w_{1} D_{x}^{2}+2 u_{1} D_{x} D_{z}\right)\right) f . f=0 .
\end{aligned}
$$

The D-operator [13] is defined by

$$
\begin{aligned}
D_{x}^{k} D_{y}^{m} D_{z}^{n} D_{t}^{l} f(x, y, z, t) \cdot g(x, y, z, t)= & \left(\frac{\partial}{\partial x}-\frac{\partial}{\partial x^{\prime}}\right)^{k}\left(\frac{\partial}{\partial y}-\frac{\partial}{\partial y^{\prime}}\right)^{m}\left(\frac{\partial}{\partial z}-\frac{\partial}{\partial z^{\prime}}\right)^{n} \\
& \left.\left(\frac{\partial}{\partial t}-\frac{\partial}{\partial t^{\prime}}\right)^{l}\left[f(x, y, z, t) g\left(x^{\prime}, y^{\prime}, z^{\prime}, t^{\prime}\right)\right]\right|_{x^{\prime}=x, y^{\prime}=y, z^{\prime}=z, t^{\prime}=t}
\end{aligned}
$$

where $f(x, y, z, t)$ is an auxiliary function. 


\section{Lump solitons FOR $(3+1)$-DIMENSIONAL BuRgERS-LIKE EQUATION}

The $(3+1)$-dimensional Burgers-like equation has a class of the positive quadratic function, the lump solutions can be expressed in the form

$$
\begin{aligned}
& f(x, y, z, t)=g^{2}+h^{2}+a_{6}, \\
& g=a_{1} x+a_{2} y+a_{3} z+a_{4} t+a_{5}, \\
& h=b_{1} x+b_{2} y+b_{3} z+b_{4} t+b_{5} .
\end{aligned}
$$

Where the parameters involved in (3.1) are arbitrary parameters to be determined later. $f$ must be positive and $u, v$ and $w$ must be localized and decay algebraically.

Plugging (3.1) into (2.2) and with the aid of Maple, some of a class of explicit solutions can be obtained as follows

$$
\begin{array}{cc}
a_{1}=0, & a_{2}=-b_{2}, \\
\alpha=\frac{-\beta b_{2}+\gamma b_{3}}{b_{1}}, & u_{1}=0,
\end{array}
$$

where $a_{i}, i=3,5,6$ and $b_{j}, j=1,2,3,4,5$ are real constants.

Then the lump solutions for $(3+1)$-dimensional Burgers-like equation (1.1) has the form

$$
\begin{aligned}
& u(x, y, z, t)=2 \frac{\left(b_{4} t+b_{1} x+b_{2} y+b_{3} z+b_{5}\right) b_{1}}{\left(-b_{2} y+a_{3} z+a_{5}\right)^{2}+\left(b_{4} t+b_{1} x+b_{2} y+b_{3} z+b_{5}\right)^{2}+a_{6}}+u_{1}, \\
& v(x, y, z, t)=\frac{-2\left(-b_{2} y+a_{3} z+a_{5}\right) b_{2}+2\left(b_{4} t+b_{1} x+b_{2} y+b_{3} z+b_{5}\right) b_{2}}{\left(-b_{2} y+a_{3} z+a_{5}\right)^{2}+\left(b_{4} t+b_{1} x+b_{2} y+b_{3} z+b_{5}\right)^{2}+a_{6}}+v_{1}, \\
& w(x, y, z, t)=\frac{2\left(-b_{2} y+a_{3} z+a_{5}\right) a_{3}+2\left(b_{4} t+b_{1} x+b_{2} y+b_{3} z+b_{5}\right) b_{3}}{\left(-b_{2} y+a_{3} z+a_{5}\right)^{2}+\left(b_{4} t+b_{1} x+b_{2} y+b_{3} z+b_{5}\right)^{2}+a_{6}}+w_{1} .
\end{aligned}
$$

The critical points for (3.1) can be given as follows

$$
\begin{aligned}
& x(y, t)=\frac{t a_{3} b_{4}-t a_{4} b_{3}-y a_{2} b_{3}+y a_{3} b_{2}+a_{3} b_{5}-a_{5} b_{3}}{a_{1} b_{3}-a_{3} b_{1}}, \\
& z(x, t)=-\frac{t a_{1} b_{4}-t a_{4} b_{1}+y a_{1} b_{2}-y a_{2} b_{1}+a_{1} b_{5}-a_{5} b_{1}}{a_{1} b_{3}-a_{3} b_{1}} .
\end{aligned}
$$

Under the condition

$$
a_{1} b_{3}-a_{3} b_{1} \neq 0
$$

at any arbitrary fixed time $t$. Also (3.4) is a critical points for $u, v$ and $w$. It is noted that at these critical points (3.4), the function $f(x, y, z, t)-a_{6}$ vanishes. This means that $f(x, y, z, t)>0$ if and only if $a_{6}>0$, which implies that $u, v$ and $w$ in (2.1) are analytical functions if and only if $a_{6}>0$.

In Figure 1a, the density propagation process for the wave $u$ and 3D-plots were shown for the lump waves which contain the shapes and the amplitudes of lump solutions for some parameters (3.2) along $x-z$ plane with $x=-10 . .10, z=-10 . .10$ individually at $y=0$ for different time $t=-10, t=0$ and $t=10$. We show that 


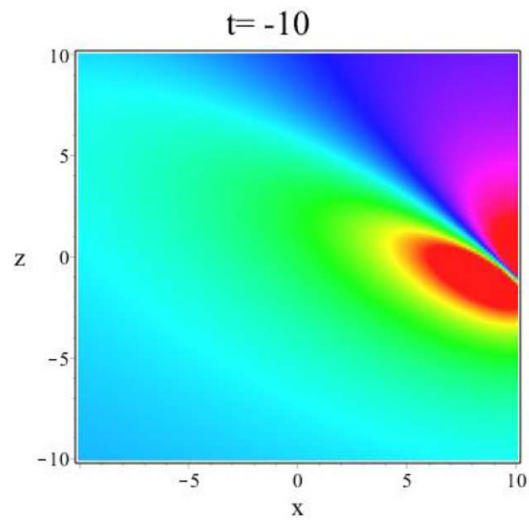

$\mathrm{t}=-10$

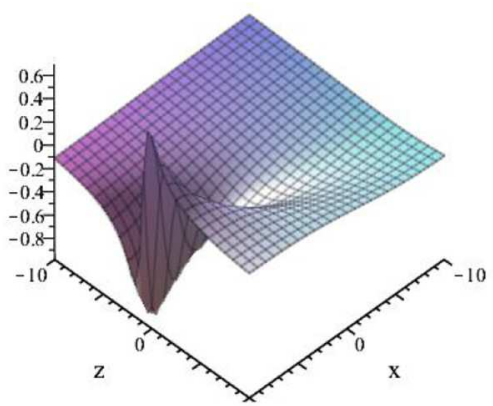

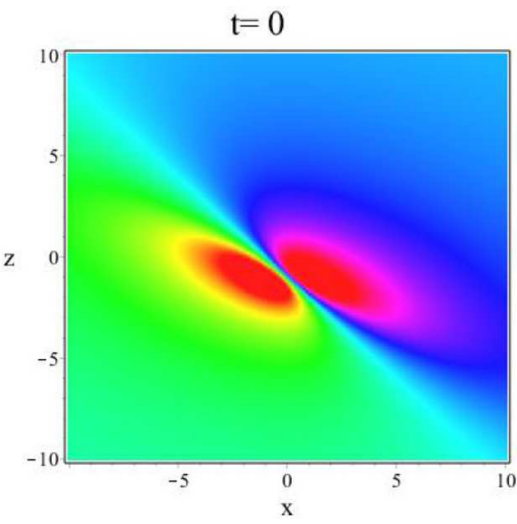

$\mathrm{t}=0$

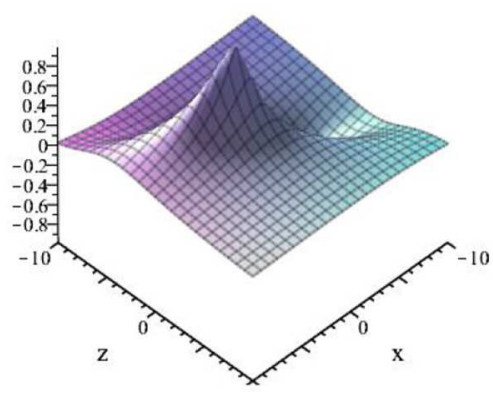

(a)

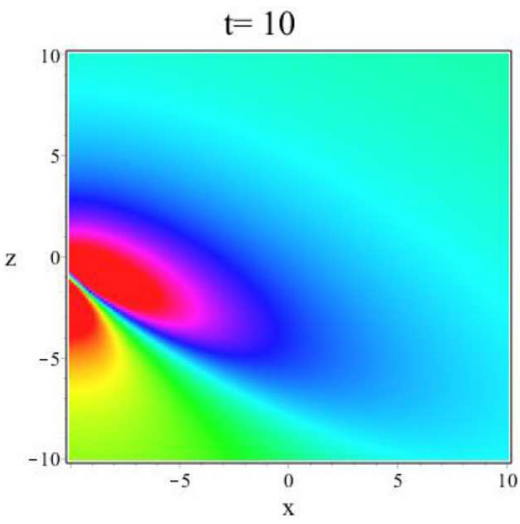

$\mathrm{t}=10$

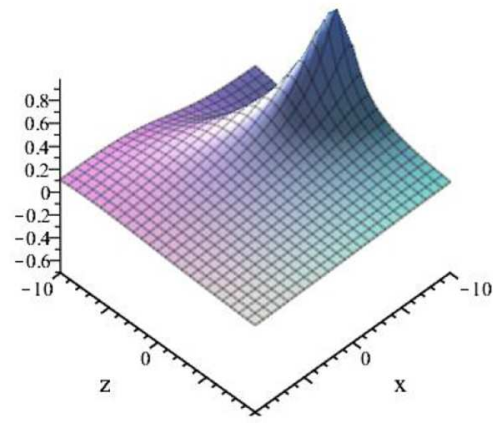

Figure 1. (a) Density and 3D plots for the lump solitons for a wave $u$ for (1.1) at $\mathrm{t}=-10,0,10$ respectively. (b) Density and 3D plots for the lump solitons for a wave $v$ for (1.1) at $\mathrm{t}=-10,0,10$ respectively. (c) Density and 3D plots for the lump solitons for a wave $w$ for (1.1) at $\mathrm{t}=-10,0,10$ respectively.

the peaks are located at the minimum point

$$
\left(\frac{-10 a_{3} b_{4}+a_{3} b_{5}+10 a_{4} b_{3}-a_{5} b_{3}}{a_{1} b_{3}-a_{3} b_{1}}, 0,-\frac{-10 a_{1} b_{4}+a_{1} b_{5}+10 a_{4} b_{1}-a_{5} b_{1}}{a_{1} b_{3}-a_{3} b_{1}}\right)
$$

and maximum point

$$
\left(\frac{10 a_{3} b_{4}+a_{3} b_{5}-10 a_{4} b_{3}-a_{5} b_{3}}{a_{1} b_{3}-a_{3} b_{1}}, 0,-\frac{10 a_{1} b_{4}+a_{1} b_{5}-10 a_{4} b_{1}-a_{5} b_{1}}{a_{1} b_{3}-a_{3} b_{1}}\right) .
$$

This means that the center of peaks and valley lies on the straight line $y=0$.

Then we can deduce that the lump solutions for any given time $t$ satisfy

$$
\lim _{t \rightarrow \pm \infty} u(x, y, z, t)=u_{1}, \quad \lim _{t \rightarrow \pm \infty} v(x, y, z, t)=v_{1}, \quad \lim _{t \rightarrow \pm \infty} w(x, y, z, t)=w_{1} .
$$



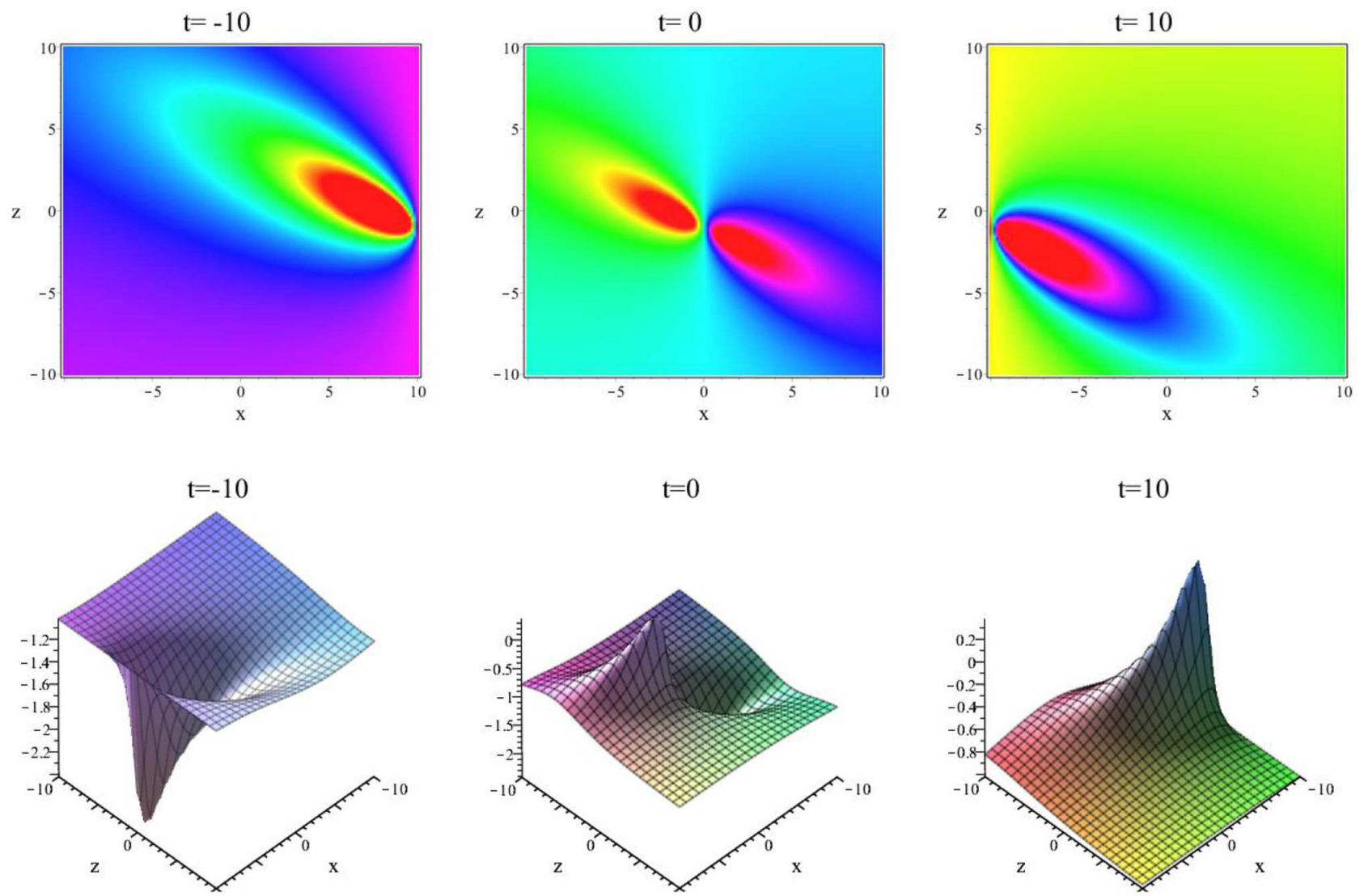

$\mathrm{t}=10$

(b)

Figure 1. Continued.

For a certain selections for the parameters in (3.1) $\alpha=2, \beta=\gamma=a_{3}=a_{5}=a_{6}=b_{1}=b_{2}=b_{3}=b_{4}=b_{5}=$ $1, a_{1}=a_{4}=0$, we can get the positive quadratic function and lump solution for the $(3+1)$-dimensional Burgers-like equation (1.1)

$$
\begin{gathered}
f(x, y, z, t)=(1-y+z)^{2}+(x+y+z+t+1)^{2}+1, \\
u(x, y, z, t)=\frac{2 x+2 y+2 z+2 t+2}{(1-y+z)^{2}+(x+y+z+t+1)^{2}+1}+u_{1}, \\
v(x, y, z, t)=\frac{4 y+2 x+2 t}{(1-y+z)^{2}+(x+y+z+t+1)^{2}+1}+v_{1}, \\
w(x, y, z, t)=\frac{4+4 z+2 x+2 t}{(1-y+z)^{2}+(x+y+z+t+1)^{2}+1}+w_{1} .
\end{gathered}
$$

It was noticed that the soliton is induced by the lump itself. A special dispersive relation is found for the soliton being determined by the lump. If the lump does not exist, the soliton will also disappear. 


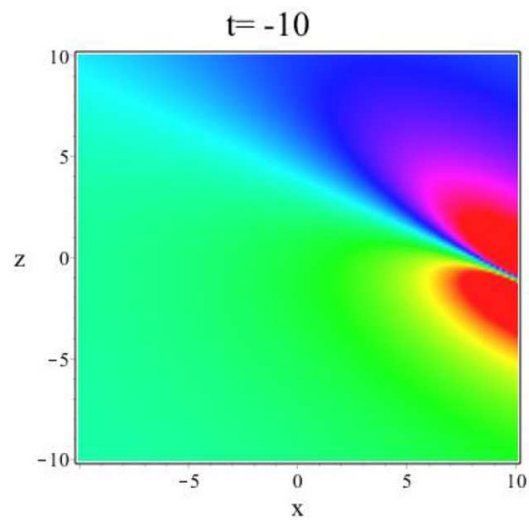

$\mathrm{t}=-10$
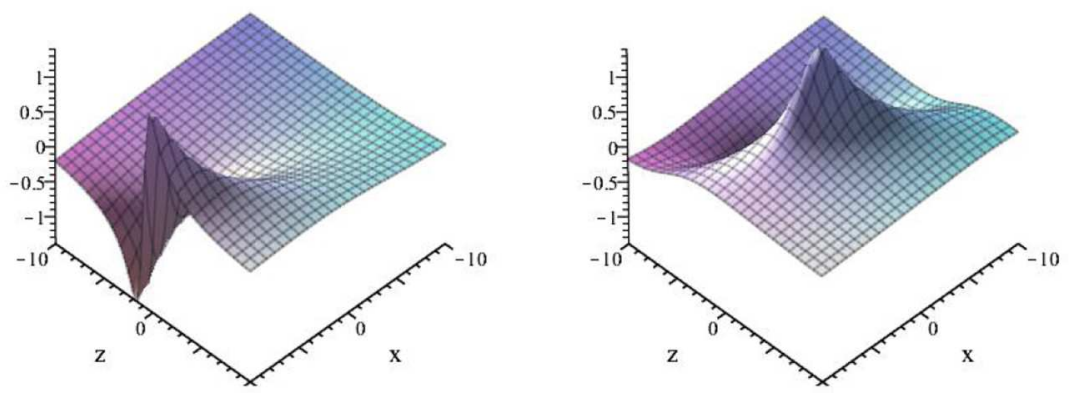

(c)
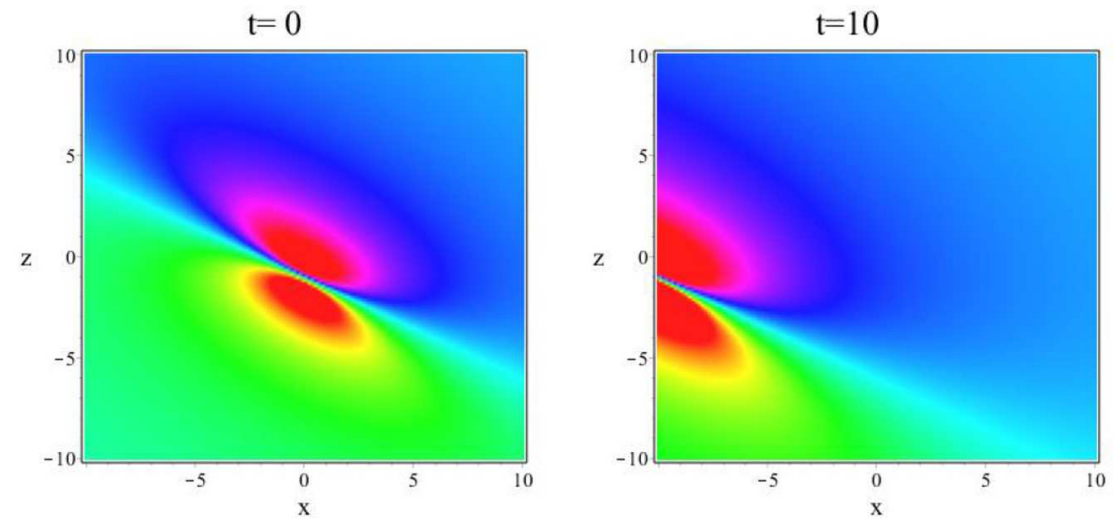

$\mathrm{t}=0$

$\mathrm{t}=10$

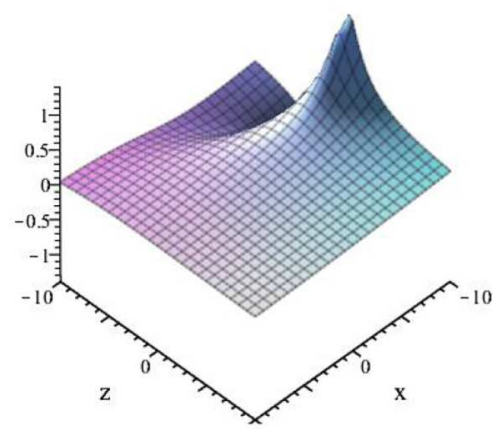

Figure 1. Continued.

\section{Rogue wave solutions for the $(3+1)$-Dimensional \\ BURGERS-LIKE EQUATION}

In this section, with the help of the bilinear form (2.2), we obtain the algebraic solitary wave solution for the $(3+1)$-dimensional Burgers-like equation (1.1) by inserting the

$$
f(x, y, z, t)=1+\left(a_{1} x+a_{2} y+a_{3} t+a_{4} z\right)^{2}+b_{1} x^{2}+b_{2} y^{2}+b_{3} t^{2}+b_{4} z^{2},
$$

where $a_{i}, b_{i}, i=1 . .4$ are real parameters. Inserting equation (4.1) into (2.2), we get an algebraic system of $x, y, z$ and $t$. Solving it with the aid of Maple, one of these solutions

$$
\begin{aligned}
& a_{3}=\beta a_{2}+\nu a_{4}, \quad b_{1}=-\frac{\left(\alpha a_{1}+\beta a_{2}+\nu a_{4}\right) a_{1}}{\alpha}, \\
& b_{2}=-2 \frac{a_{2}\left(\alpha a_{1}+\beta a_{2}+\nu a_{4}\right)}{\beta}, \quad b_{3}=-2\left(\beta a_{2}+\nu a_{4}\right)\left(\alpha a_{1}+\beta a_{2}+\nu a_{4}\right), \\
& b_{4}=-2 \frac{a_{4}\left(\alpha a_{1}+\beta a_{2}+\nu a_{4}\right)}{\nu},
\end{aligned}
$$


where $a_{i}, i=1,2,4,5$ and $b_{5}$ are constant. Then the rogue solutions for $(3+1)$-dimensional Burgers-like equation (1.1) can be obtained a follows

$$
\begin{aligned}
& u(x, y, z, t)=\frac{2\left(a_{3} t+a_{1} x+a_{2} y+a_{4} z\right) a_{1}+2 b_{1} x}{1+\left(a_{3} t+a_{1} x+a_{2} y+a_{4} z\right)^{2}+b_{1} x^{2}+b_{2} y^{2}+b_{3} t^{2}+b_{4} z^{2}}+u_{1}, \\
& v(x, y, z, t)=\frac{2\left(a_{3} t+a_{1} x+a_{2} y+a_{4} z\right) a_{2}+2 b_{2} y}{1+\left(a_{3} t+a_{1} x+a_{2} y+a_{4} z\right)^{2}+b_{1} x^{2}+b_{2} y^{2}+b_{3} t^{2}+b_{4} z^{2}}+v_{1}, \\
& w(x, y, z, t)=\frac{2\left(a_{3} t+a_{1} x+a_{2} y+a_{4} z\right) a_{4}+2 b_{4} z}{1+\left(a_{3} t+a_{1} x+a_{2} y+a_{4} z\right)^{2}+b_{1} x^{2}+b_{2} y^{2}+b_{3} t^{2}+b_{4} z^{2}}+w_{1} .
\end{aligned}
$$

The asymptotic behaviors for the solution (4.3) can be obtained

$$
\lim _{(x, y, z, t)-> \pm \infty}(u(x, y, z, t), v(x, y, z, t), w(x, y, z, t))=\left(u_{1}, v_{1}, w_{1}\right) .
$$

The maximum values for the functions $u(x, y, z, t), v(x, y, z, t)$ and $w(x, y, z, t)$ at $t=0$ are $\left.M_{u}\left( \pm \sqrt{\frac{1}{a_{4}^{2}+b_{4}}}, 0,0\right), M_{v}\left(0, \pm \sqrt{\frac{1}{a_{4}^{2}+b_{4}}}, 0,0\right), 0\right)$ and $\left.M_{w}\left(0,0, \pm \sqrt{\frac{1}{a_{4}^{2}+b_{4}}}, 0,0\right)\right)$, the maximum and minimum values for $u, v$ and $w$ are

$$
\begin{array}{ll}
u_{\min }=-\sqrt{a_{1}^{2}+b_{1}}+u_{1}, & u_{\max }=\sqrt{a_{1}^{2}+b_{1}}+u_{1}, \\
v_{\min }=-\sqrt{a_{2}^{2}+b_{2}}+v_{1}, & v_{\max }=\sqrt{a_{2}^{2}+b_{2}}+v_{1}, \\
w_{\min }=-\sqrt{a_{4}^{2}+b_{4}}+w_{1}, & w_{\max }=\sqrt{a_{4}^{2}+b_{4}}+w_{1} .
\end{array}
$$

Then the amplitude of the rogue wave with solutions (4.2) for $u, v$ and $w$ depends on nine parameters $a_{1}, b_{1}, u_{1}, a_{2}, b_{2}, v_{1}, a_{4}, b_{4}$ and $w_{1}$.

The rogue solution to the $(3+1)$-dimensional Burgers-like equation (1.1) for the special choice of parameters $a_{1}=a_{2}=a_{5}=u_{1}=v_{1}=w_{1}=1, a_{3}=2, b_{1}=-3, b_{2}=-6, b_{3}=-12, b_{4}=-6$ and $b_{5}=0$ can be expressed as

$$
\begin{aligned}
& u(x, y, z, t)=\frac{4 x-2 y-4 t-2 z}{2 x^{2}+(-2 y-4 t-2 z) x+8 t^{2}+(-4 y-4 z) t+5 y^{2}-2 z y+5 z^{2}-1}+1, \\
& v(x, y, z, t)=\frac{-2 x+10 y-4 t-2 z}{2 x^{2}+(-2 y-4 t-2 z) x+8 t^{2}+(-4 y-4 z) t+5 y^{2}-2 z y+5 z^{2}-1}+1, \\
& w(x, y, z, t)=\frac{-2 x-2 y-4 t+10 z}{2 x^{2}+(-2 y-4 t-2 z) x+8 t^{2}+(-4 y-4 z) t+5 y^{2}-2 z y+5 z^{2}-1}+1 .
\end{aligned}
$$

Rogue waves are isolated huge wave corresponding to large-amplitude waves that seldom appear on the ocean surfaces. Such waves can be accompanied by deep troughs (holes), which occur before and/or after the largest crest, these waves is a kind of dynamical phenomenon localized in both time and space. In Figure 2a, the density propagation process for the wave $u$ and 3D-plots were shown for the rogue wave solutions associated with the solutions (4.6) for $u(x, y, 1,0), v(x, y, 1,0)$ and $w(x, y, 1,0)$ respectively for the $(3+1)$-dimensional Burgers-like equation (1.1), we also noticed that these solutions are localized in both $x, y$ plane.

Also Figure 2b and the first two figures in Figure 2c show that the rogue wave have one peak which is higher than the water level. This kind is called lump wave that maintains the localization property in the planes $(x, y)$. The rogue wave excited from the bright soliton pair. From the whole evolution, the fundamental rogue wave is the rational wave. 


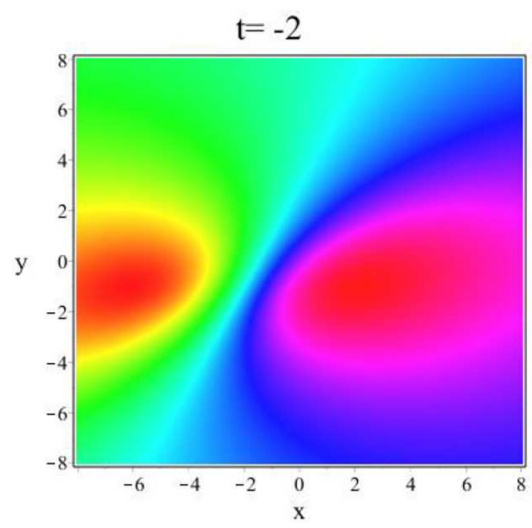

$\mathrm{t}=-2$

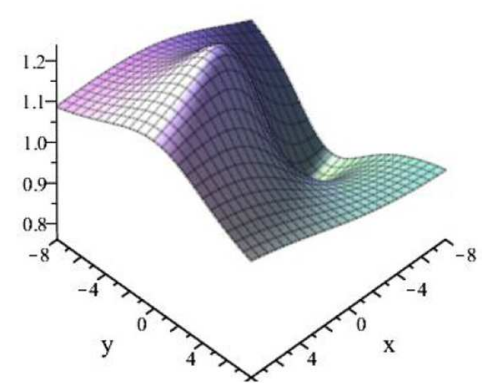

$\mathrm{t}=0$

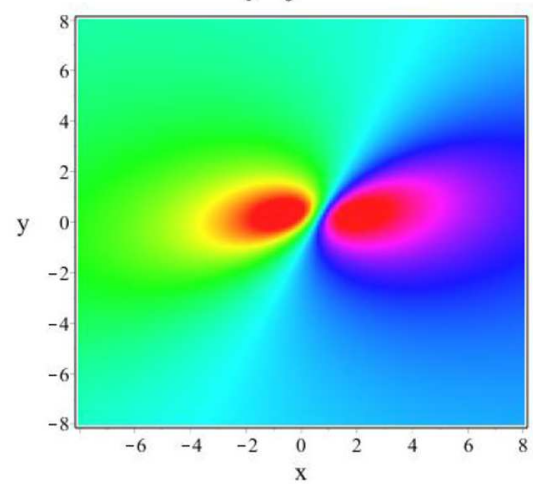

$\mathrm{t}=0$

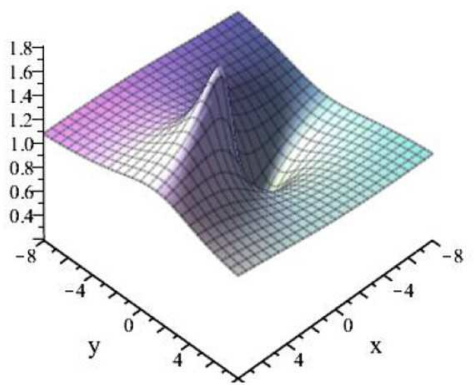

(a)

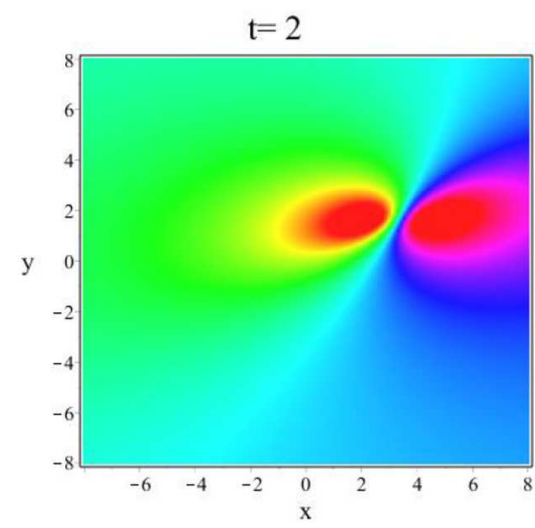

$\mathrm{t}=2$

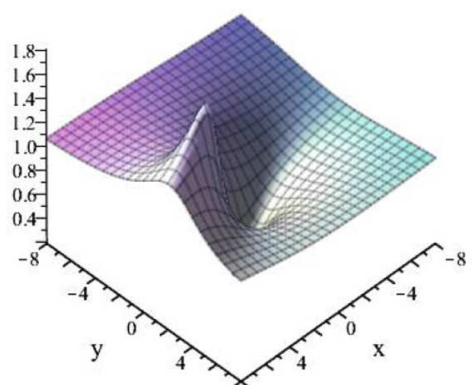

FIGURE 2. (a) Density and 3D plots for the rogue solitons for a wave $u$ for (4.6) at $t=-2,0,2$ respectively. (b) Density and 3D plots for the rogue solitons for a wave $v$ for $(4.6)$ at $t=-2,0,2$ respectively. (c) Density and $3 \mathrm{D}$ plots for the rogue solitons for a wave $w$ for $(4.6)$ at $t=-2,0,2$ respectively.

\section{Interaction Phenomena for the $(3+1)$-Dimensional BURGERS-LIKE EQUATION}

In this section, we will study the completely non-elastic interaction phenomena between a lump and a stripe or line soliton for the $(3+1)$-dimensional Burgers-like equation (1.1) by taking the function $f$ in equation $(2.2)$ in the form

$$
\begin{aligned}
f(x, y, z, t)= & \left(a_{1} x+a_{2} y+a_{3} z+a_{4} t+a_{5}\right)^{2}+\left(b_{1} x+b_{2} y+b_{3} z+b_{4} t+b_{5}\right)^{2} \\
& +a_{6}+l \exp \left(k_{1} x+k_{2} y+k_{3} t+k_{4} z\right)
\end{aligned}
$$

where $a_{i}, i=1 . .6, b_{j}, j=1 . .5, k_{n}, n=1 . .4$ and $l$ are real parameters will be determined. It is noticed that the function $f$ is a combination of a rational and an exponential function.

By substituting (5.1) into (2.2), we obtain a system of an algebraic equations, with the aid of Maple, the 


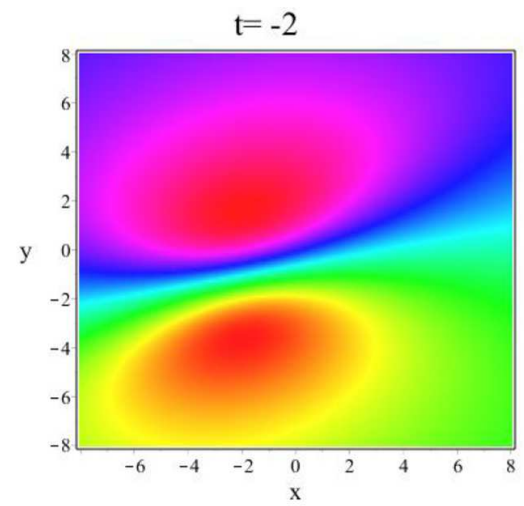

$\mathrm{t}=-2$

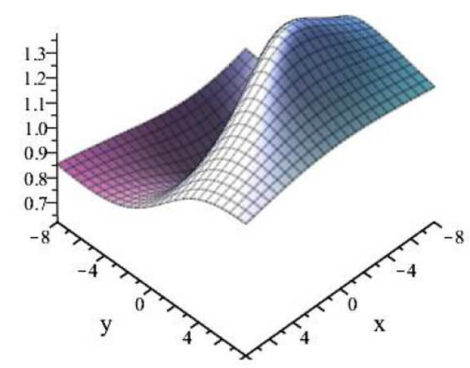

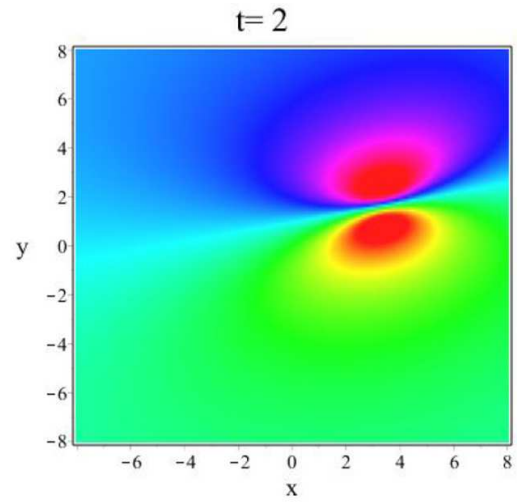

$\mathrm{t}=0$

$\mathrm{t}=2$

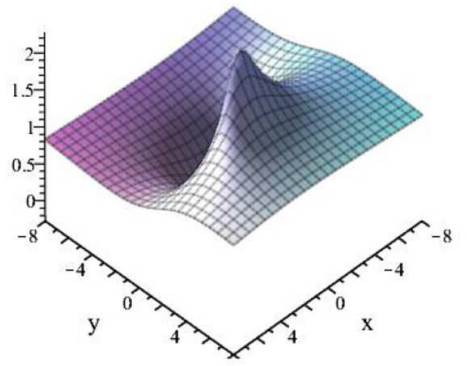

(b)

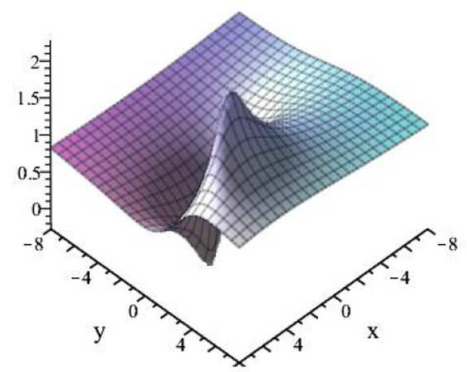

Figure 2. Continued.

following parameters in (5.1) as follows

$$
\begin{aligned}
& a_{3}=\frac{-\alpha a_{1}^{2}-\alpha b_{1}^{2}-\beta a_{1} a_{2}-\beta b_{1} b_{2}-\nu b_{1} b_{3}}{\nu a_{1}}, \\
& a_{4}=\frac{\left(-\alpha u_{1}-\beta v_{1}-\nu w_{1}\right) a_{1}^{2}+b_{1} u_{1}\left(\alpha b_{1}+\beta b_{2}+\nu b_{3}\right)}{a_{1}}, \\
& b_{4}=\left(-2 \alpha u_{1}-\beta v_{1}-\nu w_{1}\right) b_{1}-u_{1}\left(\beta b_{2}+\nu b_{3}\right), \\
& k_{3}=-\alpha k_{1}^{2}+\left(-2 \alpha u_{1}+\left(-k_{2}-v_{1}\right) \beta-\nu\left(k_{4}+w_{1}\right)\right) k_{1}-u_{1}\left(\beta k_{2}+\nu k_{4}\right),
\end{aligned}
$$

where $a_{i}, i=1,2,5,6, b_{j}, j=1,2,3$ and $k_{m}, m=1,2,4$ are constants.

The solutions for the wave $u, v$ and $w$ for the $(3+1)$-dimensional Burgers-like equation (1.1) by inserting (5.2) into the (5.1) and using (2.2) can be get as follows for special selection parameters

$$
\begin{aligned}
u(x, y, z, t) & =\frac{8 x+4 y+12 z+8 t+4+\mathrm{e}^{x+y+5 t+z}}{(2 x+y+3 z+2 t+1)^{2}+(1+y+z)^{2}+1+\mathrm{e}^{x+y+5 t+z}}+1, \\
v(x, y, z, t) & =\frac{4 x+4 y+8 z+4 t+4+\mathrm{e}^{x+y+5 t+z}}{(2 x+y+3 z+2 t+1)^{2}+(1+y+z)^{2}+1+\mathrm{e}^{x+y+5 t+z}}+1 \\
w(x, y, z, t) & =\frac{12 x+8 y+20 z+12 t+8+\mathrm{e}^{x+y+5 t+z}}{(2 x+y+3 z+2 t+1)^{2}+(1+y+z)^{2}+1+\mathrm{e}^{x+y+5 t+z}}+1 .
\end{aligned}
$$




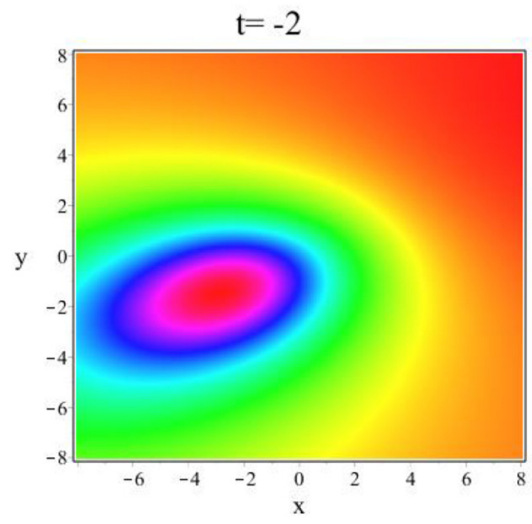

$\mathrm{t}=-2$

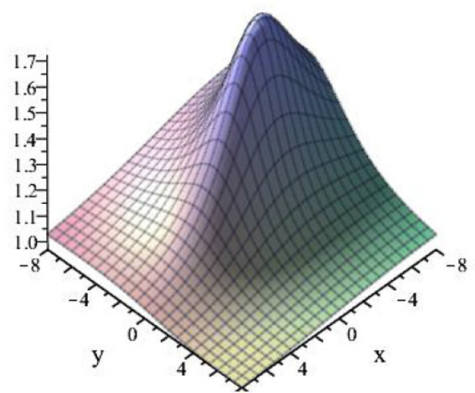

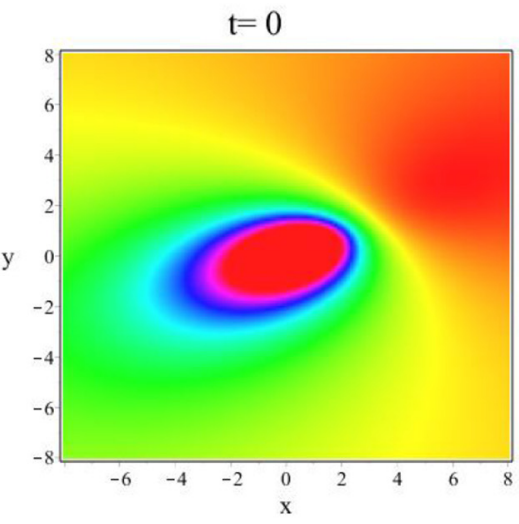

$\mathrm{t}=0$

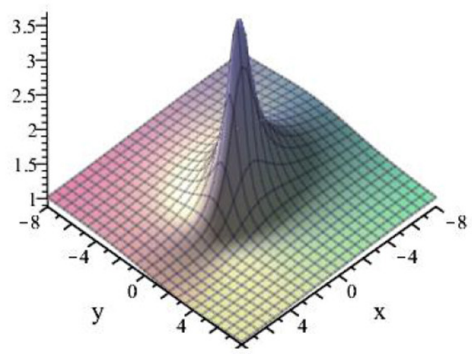

(c)
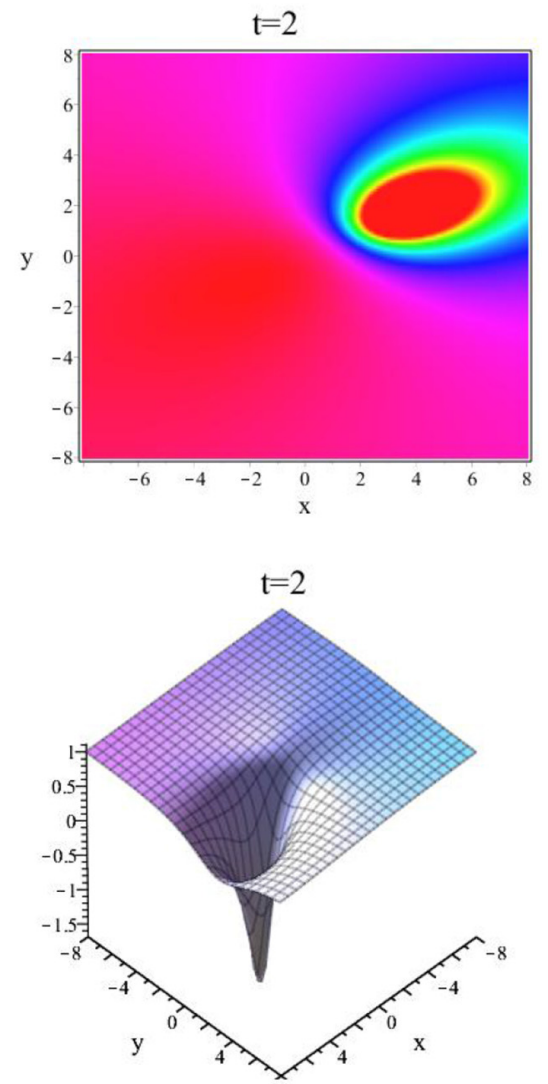

Figure 2. Continued.

The asymptotic behaviors for the solution (5.3) can be obtained

$$
\begin{aligned}
& \lim _{(x, y, z, t)->-\infty}(u(x, y, z, t), v(x, y, z, t), w(x, y, z, t))=\left(u_{1}, v_{1}, w_{1}\right), \\
& \lim _{(x, y, z, t)->+\infty}(u(x, y, z, t), v(x, y, z, t), w(x, y, z, t))=\left(k_{1}+u_{1}, k_{2}+v_{1}, k_{4}+w_{1}\right) .
\end{aligned}
$$

We note that the solution (5.3) is a mixed exponential-algebraic solitary wave solution or a rational kink solitary wave solution, which is decaying algebraically and exponentially.

One stripe soliton can split into a lump and a stripe soliton over time, this is a similar to the fission phenomenon [37]. Also, from (5.1) that $f$ can divide into three functions $f_{1}=1, f_{2}=\left(a_{1} x+a_{2} y+a_{3} z+a_{4} t+\right.$ $\left.a_{5}\right)^{2}+\left(b_{1} x+b_{2} y+b_{3} z+b_{4} t+b_{5}\right)^{2}$ and $f_{3}=l \exp \left(k_{1} x+k_{2} y+k_{3} t+k_{4} z\right)$, which also solutions for $(3+1)$ dimensional Burgers-like equation (1.1), so we can form a solitary wave from any combinations $\left(f_{1}, f_{2}\right),\left(f_{2}, f_{3}\right)$ and $\left(f_{1}, f_{3}\right)$ a follows

$$
\begin{aligned}
& S=1+\mathrm{e}^{x+y+5 t+z}, \\
& L=1+(2 x+y+3 z+2 t+1)^{2}+(1+y+z)^{2}, \\
& S-L=1+(2 x+y+3 z+2 t+1)^{2}+(1+y+z)^{2}+\mathrm{e}^{x+y+5 t+z} .
\end{aligned}
$$




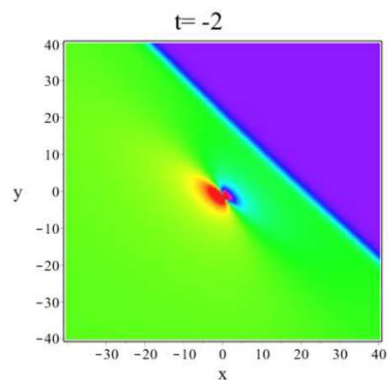

$t=-2$

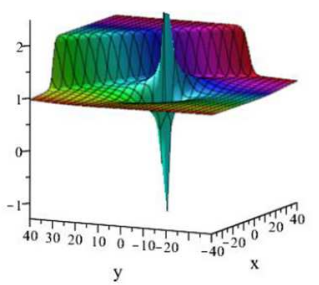

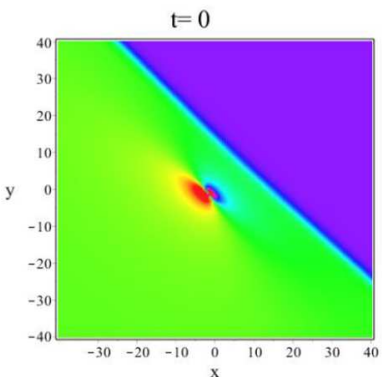

$\mathrm{t}=0$

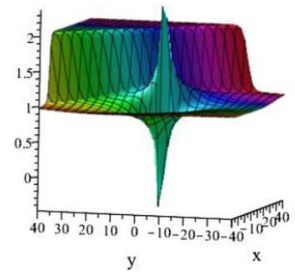

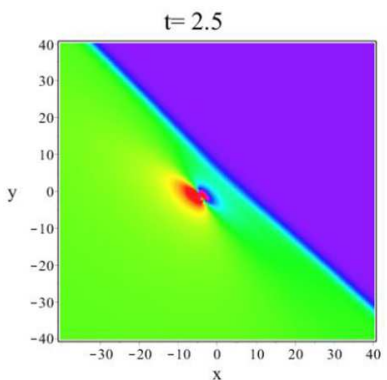

$\mathrm{t}=2.5$

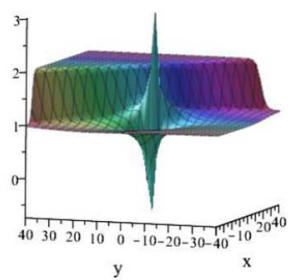

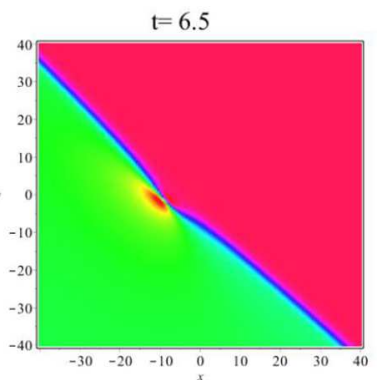

$\mathrm{t}=6.5$

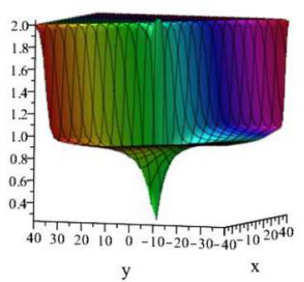

(a)

FiguRE 3. (a) Density and 3D plots for the lump-stripe solitons for a wave $u$ for (5.3) at $\mathrm{t}=$ $-2,0,2.5$ and 6.5 respectively. (b) Density and 3D plots for the lump-stripe solitons for a wave $v$ for (5.3) at $\mathrm{t}=-2,0,2.5$ and 6.5 respectively. (c) Density and 3D plots for the lump-stripe solitons for a wave $w$ for $(5.3)$ at $\mathrm{t}=-2,0,2.5$ and 6.5 respectively.

$S$ is a single-kink solitary wave, $L$ is a lump soliton solution and $S-L$ is a lump-stripe solutions for $(3+1)$-dimensional Burgers-like equation (1.1).

The lump and a stripe soliton start appearing at time $t=-2$ in Figure 3a, the stripe soliton moves through the times $t=0,2.5,6.5$ until it completely disappears, this proves that the interaction between lump and stripe soliton is completely non-elastic. This interaction phenomena is similar to the fusion and fission for soliton solutions.

\section{Discussions AND CONClusions}

In summary, we successfully derived the Hirota bilinear form via a direct method and multiple-kink solutions form for $(3+1)$-dimensional Burgers-like equation. Also, we investigated lump solitons and rogue wave solutions for this equation via the bilinear method, these solutions are non-singular and localized and shown by density and $3 \mathrm{D}$ plots for a special parameters solution by Maple. Completely non-elastic interaction lump-stripe (lumpoff) solitons for suitable choice of parameters in (5.3) are obtained that is, in the beginning at $t=-2$ lump soliton are separated from the stripe soliton at times $t=0,2.56,6.5$, lump soliton begin to be swallowed gradually until disappearing. The obtained results are helpful to understand the propagation processes for nonlinear waves for $(3+1)$-dimensional Burgers-like equation (1.1). The mathematical approach can be used to looking for some other classes of lump waves, rogue waves and interaction solutions for different nonlinear models in mathematical physics. We can say that the soliton and a pair of soliton or two soliton depends on the lump wave. When lump determines a soliton, with the time evolution, the lump wave will be cut by soliton, to become lumpoff. When lump determines two soliton, it becomes rogue wave and it is only seen at a specific point in time. One can be verified that these obtained results can't be found in the previous works. 


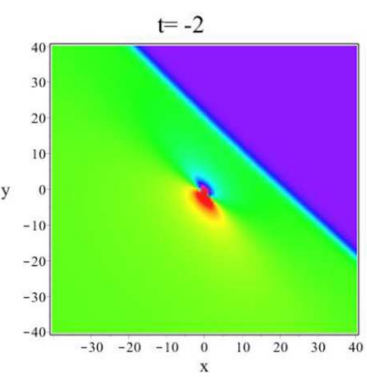

$t=-2$
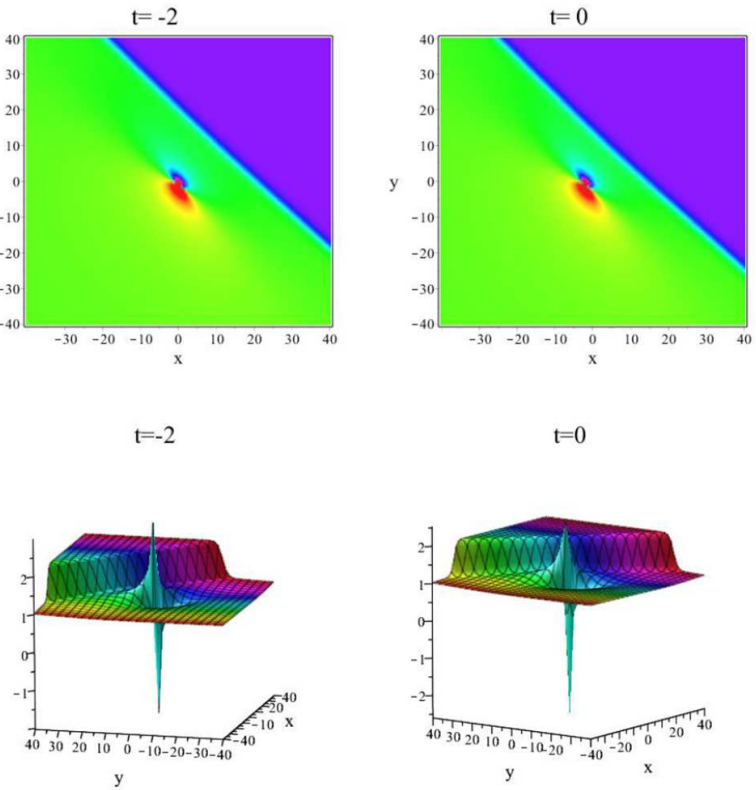

$\mathrm{t}=0$

Figure 3. Continued.

$t=-2$

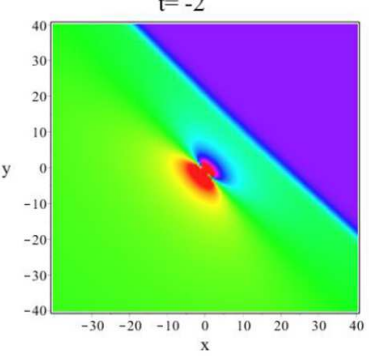

$t=-2$

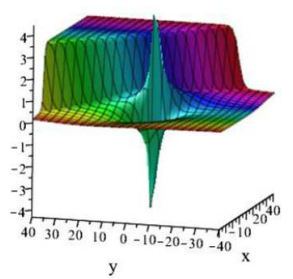

$\mathrm{t}=0$

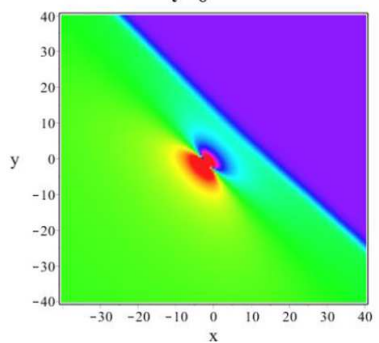

$\mathrm{t}=0$

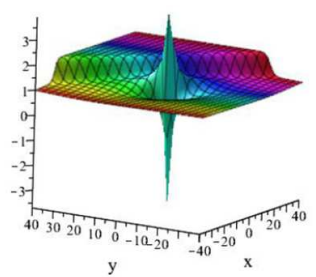

$\mathrm{t}=2.5$

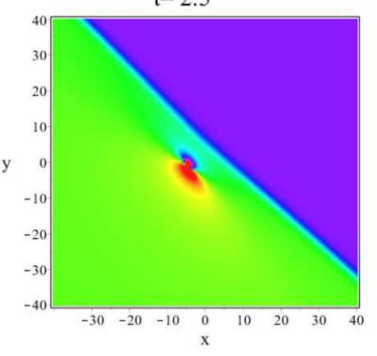

$\mathrm{t}=2.5$

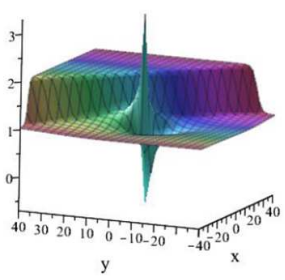

(b)

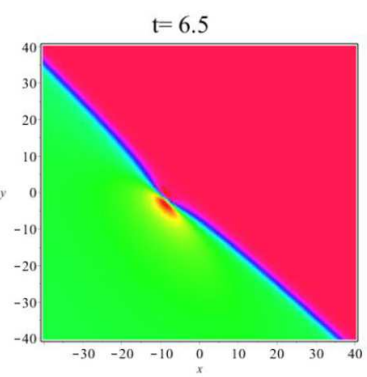

$\mathrm{t}=6.5$

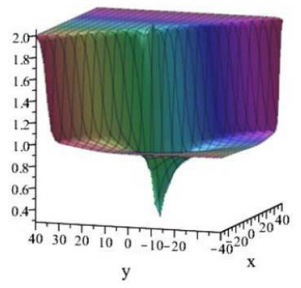

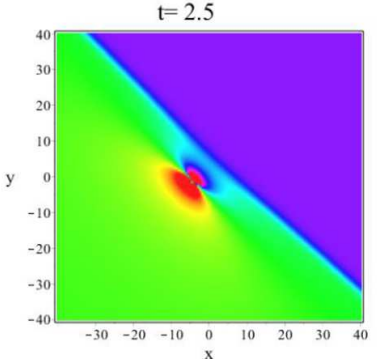

$t=2.5$

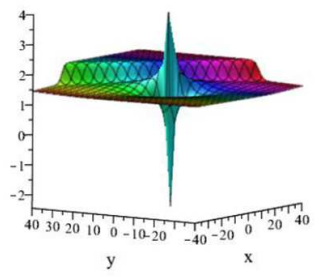

$t=6.5$

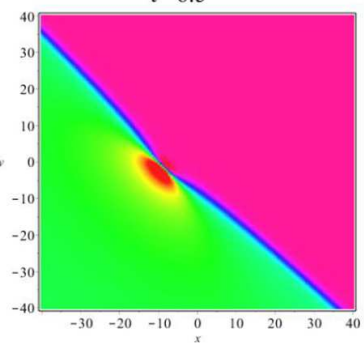

$\mathrm{t}=6.5$

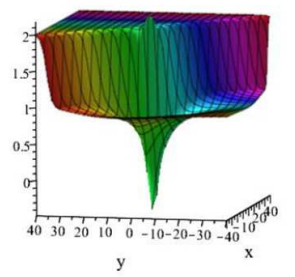

(c)

Figure 3. Continued.

\section{REFERENCES}

[1] H. Bateman, Some recent researches on the motion of fluids. Mon. Weather Rev. 43 (1915) 163-170.

[2] J.M. Burgers, A mathematical model illustrating the theory of turbulence. Adv. Appl. Mech. 1 (1948) $171-199$.

[3] F. Calogero and A. Degasperis, Spectral transform and solitons. North-Holland, Amsterdam (1982). 
[4] J.C. Chen, Y. Chen, B.F. Feng and K.I. Maruno, Multi-dark soliton solutions of the twodimensional multi-component yajimaoikawa systems. J. Phys. Soc. Jpn. 84 (2015) 034002.

[5] S.-S. Chen, B. Tian, Y. Sun and C.-R. Zhang, Generalized Darboux Transformations, Rogue Waves, and Modulation Instability for the Coherently Coupled Nonlinear Schródinger Equations in Nonlinear Optics. Ann. Phys. 531 (2019) 1900011.

[6] Z. Du, B. Tian, H.-P. Chai and X.-H. Zhao, Dark-bright semi-rational solitons and breathers for a higher-order coupled nonlinear Schródinger system in an optical fiber. Appl. Math. Lett. 102 (2020) 106110.

[7] X.-X. Du, B. Tian, Q.-X. Qu, Y.-Q. Yuan and X.-H. Zhao, Lie group analysis, solitons, self-adjointness and conservation laws of the modified Zakharov-Kuznetsov equation in an electron-positron-ion magnetoplasma. Chaos Solitons Fract. 134 (2020) 109709.

[8] P.G. Estëvez and J. Prada, Lump solutions for PDEs: algorithmic construction and classification. J. Nonlinear Math. Phys. 15 (2008) 166-175.

[9] X.-Y. Gao, Mathematical view with observational/experimental consideration on certain $(2+1)$-dimensional waves in the cosmic/laboratory dusty plasmas. Appl. Math. Lett. 91 (2019) 165-172.

[10] C. Garrett and J. Gemmrich, Rogue waves. Physics Today 62 (2009) 62-63.

[11] X.-Y. Gao, Y.-J. Guo and W.-R. Shan, Water-wave symbolic computation for the Earth, Enceladus and Titan: The higher-order Boussinesq-Burgers system, auto- and non-auto-Bácklund transformations. Appl. Math. Lett. 104 (2020) 106170.

[12] R. Hirota, Exact solution of the Korteweg-de Vries equation for multiple collisions of solitons. Phys, Rev. Lett. 27 (1971) $1192-1194$.

[13] R. Hirota, Direct method in soliton theory, in Solitons, edited by R.K. Bullough, P.J. Caudrey. Springer, Berlin, (1980).

[14] C.-C. Hu, B. Tian, H.-M. Yin, C.-R. Zhang and Z. Zhang, Dark breather waves, dark lump waves and lump wave-soliton interactions for a (3+1)- dimensional generalized Kadomtsev-Petviashvili equation in a fluid. Comput. Math. Appl. 78 (2019) $166-177$.

[15] L. Huang and Y. Chen, Lump solutions and interaction phenomenon for $(2+1)$-dimensional Sawada-Kotera equation. Theor. Phys. 67 (2017) 473-478.

[16] K. Imai, Dromion and lump solutions of the Ishimori-I equation. Progr. Theor. Phys. 98 (1997) $1013-1023$.

[17] B.-Q. Li and Y.-L. Ma, Multiple-lump waves for a (3+1)-dimensional Boiti-Leon-Manna-Pempinelli equation arising from incompressible fluid. Comput. Math. Appl. 76 (2018) 204-214.

[18] B.-Q. Li and Y.-L. Ma, Solitons resonant behavior for a waveguide directional coupler system in optical fbers. Opt. Quantum Electron. 50 (2018) 270.

[19] B.-Q. Li, Y.-L. Ma, L.-P. Mo and Y.-Y. Fu, The N-loop soliton solutions for $(2+1)$-dimensional Vakhnenko equation. Comput. Math. Appl. 74 (2017) 504-512.

[20] Z. Lu and Y. Chen, Construction of rogue wave and lump solutions for nonlinear evolution equations. Eur. Phys. J. B 88 (2015) $1-5$.

[21] Z. Lu, E.M. Tian and R. Grimshaw, Interaction of two lump solitons described by the Kadomtsev-Petviashvili I equation. Wave Motion 40 (2004) 123-135.

[22] Y.-L. Ma, Interaction and energy transition between the breather and rogue wave for a generalized nonlinear Schrödinger system with two higher-order dispersion operators in optical fibers. Nonlinear Dyn. 97 (2019) 95-105.

[23] Y.-L. Ma and B.-Q. Li, Interactions between soliton and rogue wave for a (2+1)-dimensional generalized breaking soliton system: Hidden rogue wave and hidden soliton. Comput. Math. Appl. 78 (2019) 827-839.

[24] Y.-L. Ma and B.-Q. Li, Mixed lump and soliton solutions for a generalized (3+1)-dimensional Kadomtsev-Petviashvili equation. AIMS Math. 5 (2020) 1162-1176

[25] Y.-L. Ma and B.-Q., Rogue wave solutions, soliton and rogue wave mixed solution for a generalized $(3+1)$-dimensional Kadomtsev-Petviashvili equation in fluids. Mod. Phys. Lett. B 32 (2018) 1850358.

[26] S.V. Manakov and V.E. Zakharov, Two-dimensional solitons of the Kadomtsev-Petviashvili equation and their interaction. Phys. Lett. A 63 (1977) 205-206.

[27] S. Manukure, Y. Zhou and W.X. Ma, Lump solutions to a $(2+1)$-dimensional extended KP equation. Comput. Math. Appl. 75 (2018) 2414-2419.

[28] H.E. Nistazakis, D.J. Frantzeskakis and B.A. Malomed, Collisions between spatiotemporal solitons of different dimensionality in a planar waveguide. Phys. Rev. E 64 (2001) 026604.

[29] H.-L. Si and B.-Q. Li, Two types of soliton twining behaviors for the Kraenkel-Manna-Merle system in saturated ferromagnetic materials. Optik 166 (2018) 49-55.

[30] H.-Q. Sun and A.-H. Chen, Interactional solutions of a lump and a solitary wave for two higher-dimensional equations. Nonlinear Dyn. 94 (2018) 1753-1762.

[31] Y. Tang, S. Tao and Q. Guan, Lumpsolitons and the interaction phenomena of them for two classes of nonlinear evolution equations. Comput. Math. Appl. 72 (2016) 2334-2342.

[32] X. Wang, Y.Q. Li, F. Huang and Y. Chen, Rogue wave solitons of AB system. Commun. Nonlinear Sci. Numer. Simul. 20 (2015) 434-442.

[33] C. Wang, Z. Dai and C. Liu, Interaction between kink solitary wave and rogue wave for $(2+1)$-dimensional Burgers equation. Mediterr. J. Math. 13 (2016) 1087-1098.

[34] M. Wang, B. Tian, Y. Sun and Z. Zhang, Lump, mixed lump-stripe and rogue wave-stripe solutions of a (3+1)-dimensional nonlinear. Comput. Math. Appl. 79 (2020) 576-587.

[35] A.M. Wazwaz, Partial Differential Equations and Solitary Waves Theorem. Springer and HEP, Berlin (2009). 
[36] A.-M. Wazwaz and S.A. El-Tantawy, New (3+1)-dimensional equations of Burgers type and Sharma-Tasso-Olver type: multiplesoliton solutions. Nonlinear Dyn. 87 (2017) 2457-2461.

[37] H.W. Yang, X. Chen, M. Guo and Y.D. Chen, A new ZK-BO equation for three-dimensional algebraic Rossby solitary waves and its solution as well as fission property. Nonlinear Dyn. 91 (2018) 2019-2032.

[38] H.-M. Yin, B. Tian and X.-C. Zhao, Chaotic breathers and breather fission/fusion for a vector nonlinear Schrödinger equation in a birefringent optical fiber or wavelength division multiplexed system. Appl. Math. Comput. 368 (2020) 124768.

[39] C.-R. Zhang, B. Tian, Q.-X. Qu, L. Liu and H.-Y. Tian, Vector bright solitons and their interactions of the couple Fokas-Lenells system in a birefringent optical fiber. Z. Angew. Math. Phys. 71 (2020) 18. 